\title{
Association of perinatal factors of epilepsy in very low birth weight infants, using a nationwide database in Japan
}

\author{
Yuki Matsushita ${ }^{1,2,4} \cdot$ Yasunari Sakai $^{1} \cdot$ Michiko Torio $^{1} \cdot$ Hirosuke Inoue $^{1,2} \cdot$ Masayuki Ochiai $^{3} \cdot$ Kazuaki Yasuoka $^{1,2}$. \\ Hiroaki Kurata $^{1,2} \cdot$ Junko Fujiyoshi, $^{1,2} \cdot$ Masako Ichiyama $^{1,2} \cdot$ Tomoaki Taguchi $^{2} \cdot$ Kiyoko Kato $^{2}$. \\ Shouichi Ohga ${ }^{1,2}$ on behalf of the Neonatal Research Network of Japan (NRNJ)
}

Received: 29 December 2018 / Revised: 27 July 2019 / Accepted: 5 August 2019 / Published online: 16 September 2019

(c) The Author(s) 2019. This article is published with open access

\begin{abstract}
Objective To determine clinical features of very low birth weight infants (VLBWIs) who had developed epilepsy by age 3 years.

Study design Multicenter cohort study using the Neonatal Research Network of Japan database. We analyzed clinical variables of 8431 VLBWIs who had recorded data of neurological sequelae at age 3 years. Logistic regression identified the association between variables and development of epilepsy.

Result One hundred and forty-three (1.7\%) infants developed epilepsy, 683 (8.1\%) showed cerebral palsy (CP), and 1114 $(13.2 \%)$ had psychomotor delay. Epilepsy was associated with history of sepsis [adjusted odds ratio (AOR) 3.23], severe intraventricular hemorrhage (IVH; AOR 5.13), and cystic periventricular leukomalacia (PVL; AOR 12.7). Severe IVH and cystic PVL were also frequently associated with CP and psychomotor delay.

Conclusion Severe IVH and cystic PVL are strongly associated with development of epilepsy, as well as other neurological sequelae, and are potential critical therapeutic targets.
\end{abstract}

\section{Introduction}

Advances in perinatal-neonatal medicine have improved the survival rate of very low birth weight infants (VLBWIs), preterm-delivered infants weighing $<1.5 \mathrm{~kg}$ at birth [1-4].

Supplementary information The online version of this article (https:// doi.org/10.1038/s41372-019-0494-7) contains supplementary material, which is available to authorized users.

Masayuki Ochiai

ochimasa@pediatr.med.kyushu-u.ac.jp

1 Department of Pediatrics, Graduate School of Medical Sciences, Kyushu University, Fukuoka, Japan

2 Comprehensive Maternity and Perinatal Care Center, Kyushu University, Fukuoka, Japan

3 Department of Perinatal and Pediatric Medicine, Graduate School of Medical Sciences, Kyushu University, Fukuoka, Japan

4 Present address: Department of Pediatrics, Clinical Research Institute, National Hospital Organization, Kyushu Medical Center, Fukuoka, Japan
However, multicenter cohorts in Japan have shown that $8-15 \%$ of VLBWIs have cerebral palsy (CP) or delay in psychomotor development [5]. The developing brains of VLBWIs are thus considered vulnerable to environmental stress and carry a higher risk than brains of term infants for neurological sequelae.

Increased attention has been paid to epilepsy among the various sequelae of VLBWIs because preterm infants have greater susceptibility to the development of epilepsy in their later life [6]. Large population studies have been conducted in various countries [7,8], although the prevalence of epilepsy among VLBWIs has not been precisely determined in Japan. The Neonatal Research Network of Japan (NRNJ) has conducted a nationwide cohort study of VLBWIs since 2003 [9, 10]. The NRNJ database contains all perinatal records from birth to 3-6 years of follow-up [11]. To date, however, perinatal factors associated with the development of neurological sequelae remain to be identified with high-powered studies. We therefore aimed to determine the prevalence rate and associated factors for epilepsy, as well as other neurological sequelae, in VLBWIs using the NRNJ database. 


\section{Subjects and methods}

\section{Study subjects}

A total of 16,870 VLBWIs were born in 57 neonatal intensive care units (NICUs) that participated in a follow-up study of the NRNJ from 2003 to 2012 (Fig. 1). We excluded $184(1.1 \%)$ infants who showed major congenital abnormalities: definitive chromosomal aberrancy and central nervous system disease of anencephaly, meningocele, fetal hydrocephaly and holoprosencephaly. A total of 1215 (7.2\%) VLBWIs died or moved to external institutes before their discharge from these NICUs. Among the 15,471 survivors of the perinatal period, 6912 (41.0\%) VLBWIs were excluded because they did not have recorded data for history of epilepsy. One hundred and twenty-eight $(0.8 \%)$ children died between the time of discharge and 3 years of follow-up. Thus, 8431 VLBWIs had recorded data for epilepsy and other long-term neurological sequelae at age 3 years.

\section{Clinical data and variables}

Clinical data included maternal complications and medication received during pregnancy and delivery. Independent variables were causative factors shown to affect neurological sequelae. Chorioamnionitis was diagnosed according to clinical findings when histological specimens were unavailable [12]. Antenatal corticosteroids were administered when preterm delivery was inevitable.

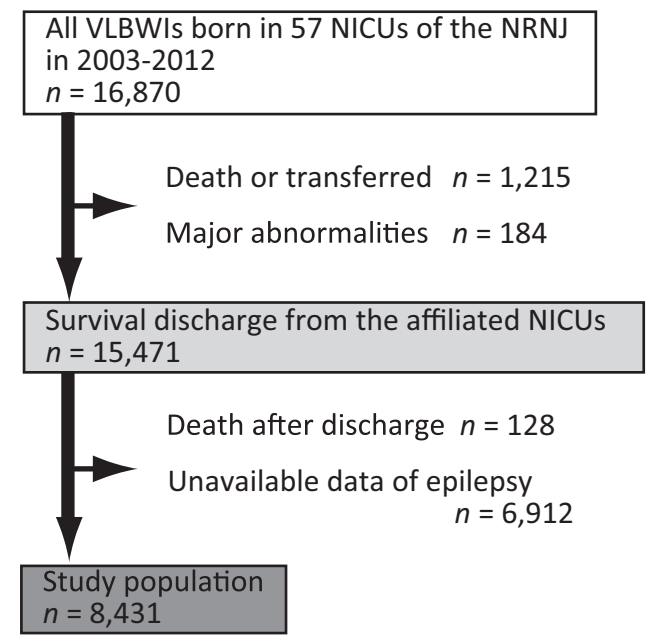

Fig. 1 Very low birth weight infants (VLBWIs) enrolled in the present study. Among 16,870 VLBWIs who were born during 2003-2012, $15,471(91.7 \%)$ were registered as survivors of the neonatal period. After discharge from hospital, $128(0.8 \%)$ died by age 3 years. Medication records or other data for epilepsy were inaccessible for $6912(40.9 \%)$ VLBWIs and these were excluded. A total of 8431 VLBWIs were registered as eligible for the present study
Gestational age was calculated from the monthly ultrasound examination and the date of the last menstrual period. Small-for-gestational age was defined as birth weight below the 10th percentile of the Japanese birth size standard [13]. Moderate-to-severe bronchopulmonary dysplasia was defined as a respiratory disturbance receiving supplemental oxygen or positive pressure at gestational age of 36 weeks [14]. Patent ductus arteriosus was diagnosed by echocardiography and was treated by indomethacin or surgical ligation, when it was symptomatic and thought to be a possible contributor of medical state. Sepsis was defined as culture-proven septicemia or bacteremia during the hospital stay. Severe intraventricular hemorrhage (IVH) was defined as grade 3 or 4 according to the scale of Papile et al. [15]. Cystic periventricular leukomalacia (PVL) was defined as periventricular cyst formation. Necrotizing enterocolitis was diagnosed according to signs of pneumoperitoneum on radiographic examination [16]. Retinopathy of prematurity was diagnosed according to the international classification [17], and was treated by the laser/cryo-coagulation or intravitreal injection of an angiogenesis inhibitor.

\section{Neurological evaluation at age 3 years}

Neurological evaluations were performed at age 3 years in accordance with a protocol of the Japanese Society for Follow-up Study of High-Risk Infants [5]. Trained testers performed developmental testing using the Kyoto Scale of Psychological Development 2001 or Enjoji Scale of Infant Analytical Development [18]. Developmental quotient (DQ) was obtained from the relative value of the developmental age to the chronological age, with the scale adjusted to $0-100$. The criterion for psychomotor delay was set to DQ <70 [5]. CP was defined as a nonprogressive central nervous system disorder characterized by abnormal muscle tone in at least one extremity and uncontrollable movements or posture [19]. Epilepsy was defined as a history of recurrent seizures or the use of antiepileptic drugs [20]. VLBWIs with a history of neonatal seizure or febrile convulsion were not counted as those with epilepsy.

\section{Statistical analyses}

All statistical analyses were performed using $\mathrm{JMP}^{\mathrm{R}}$ 11 software (SAS Institute Inc., Cary, NC, USA). Continuous and discrete variables were analyzed using the Wilcoxon rank-sum test and the chi-squared test, respectively. A multiple logistic regression model yielded odds ratios (ORs) and 95\% confidence intervals (CIs). Results with $P<0.05$ were considered statistically significant. 
Table 1 Clinical characteristics of the eligible population

\begin{tabular}{|c|c|c|c|c|c|c|c|}
\hline \multirow[b]{2}{*}{ Variables } & \multicolumn{3}{|c|}{ Study population $(n=8431)$} & \multicolumn{3}{|c|}{ Not evaluated $(n=6912)$} & \multirow[b]{2}{*}{$P$} \\
\hline & ${ }^{\mathrm{a}}$ Total & Median/n & Range/\% & ${ }^{\mathrm{a}}$ Total & Median $/ n$ & Range/\% & \\
\hline${ }^{\mathrm{b}}$ Gestational age [weeks, days] median, range & 8424 & $28 w 6 d$ & $22 w 0 d-39 w 1 d$ & 6905 & $29 \mathrm{w} 0 \mathrm{~d}$ & $21 w 2 d-40 w 1 d$ & $<0.01$ \\
\hline${ }^{\mathrm{b}}$ Birth weight [grams] median, range & 8431 & 1066 & $267-1500$ & 6911 & 1108 & $308-1500$ & $<0.01$ \\
\hline Maternal age of 35 or more years old & 7937 & 2316 & 29.2 & 6522 & 1851 & 28.4 & 0.29 \\
\hline Multiple birth & 8431 & 2128 & 25.2 & 6912 & 1748 & 25.3 & 0.94 \\
\hline Chorioamnionitis & 8345 & 2250 & 27.0 & 6796 & 1656 & 24.4 & $<0.01$ \\
\hline Antenatal steroid & 8409 & 3857 & 45.9 & 6885 & 2704 & 39.3 & $<0.01$ \\
\hline Cesarean section & 8427 & 6733 & 79.9 & 6901 & 5236 & 75.9 & $<0.01$ \\
\hline Male & 8423 & 4337 & 51.5 & 6909 & 3542 & 51.3 & 0.78 \\
\hline Apgar score of less than 7-point at 5-minutes after birth & 7914 & 1459 & 18.4 & 6353 & 1036 & 16.3 & $<0.01$ \\
\hline Small-for-gestational age & 8331 & 3333 & 40.0 & 6867 & 2621 & 38.2 & 0.02 \\
\hline Respiratory distress syndrome & 8415 & 4736 & 56.3 & 6805 & 3387 & 49.8 & $<0.01$ \\
\hline Moderate-to severe bronchopulmonary dysplasia & 3133 & 1587 & 50.7 & 2258 & 1072 & 47.5 & 0.02 \\
\hline Sepsis & 8411 & 590 & 7.0 & 6796 & 358 & 5.3 & $<0.01$ \\
\hline Symptomatic patent ductus arteriosus & 7093 & 3207 & 45.2 & 5252 & 2401 & 45.7 & 0.58 \\
\hline Severe intraventricular hemorrhage & 8425 & 231 & 2.7 & 6895 & 240 & 3.5 & 0.01 \\
\hline Cystic periventricular leukomalacia & 8424 & 255 & 3.0 & 6885 & 237 & 3.4 & 0.15 \\
\hline Necrotizing enterocolitis & 8418 & 59 & 0.7 & 6798 & 70 & 1.0 & 0.03 \\
\hline Treating retinopathy of prematurity & 8292 & 1247 & 15.0 & 6496 & 780 & 12.0 & $<0.01$ \\
\hline Cerebral palsy & 8317 & 683 & 8.2 & 2090 & 176 & 8.4 & 0.76 \\
\hline Developmental quotient of less than 70 & 7478 & 1114 & 14.9 & 1897 & 311 & 16.4 & 0.10 \\
\hline
\end{tabular}

${ }^{\mathrm{a}}$ Total number with available information

${ }^{\mathrm{b}}$ Continuous variables are expressed as the median and range

Statistically significant $P$-values are in bold italics

\section{Results}

\section{Clinical profiles of the study population}

To test whether the enrolled subjects appropriately represented the parent population of VLBWIs, we first analyzed the demographic features of VLBWIs with $(n=8431)$ or without $(n=6912)$ available data for history of epilepsy (Table 1). Significant differences were observed in 13 of 20 items of the maternal complications or perinatal histories. The proportion of VLBWIs who developed CP $(8.2 \%$ and $8.4 \%$ ) or psychomotor delay (14.9\% and $16.4 \%)$ did not differ in the rates between the two subpopulations, although those without data for epilepsy had missing data on CP and psychomotor delay. The clinical profiles of VLBWIs in our study were consistent with those in previous NRNJ studies $[9,10,18,21-24]$.

\section{Incidence of neurodevelopmental sequealae}

We determined the number of VLBWIs presenting with CP, psychomotor delay, or epilepsy among the study population $(n=8431$, Fig. 1). Overall, 1524 (18.1\%) VLBWIs had at

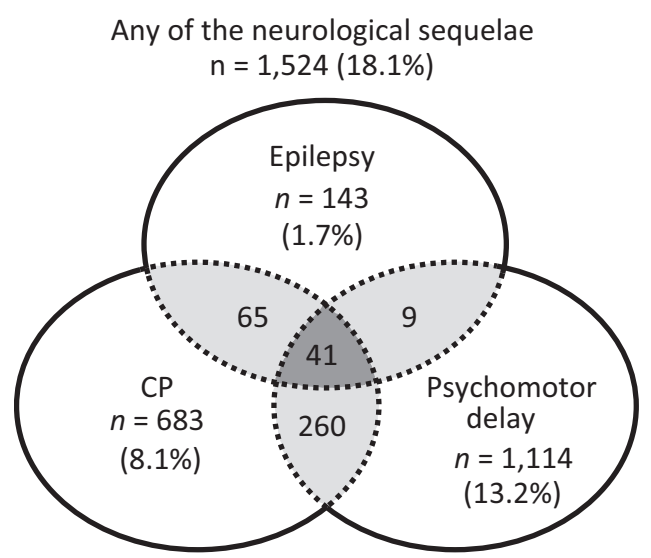

Fig. 2 Prevalence of very low birth weight infants (VLBWIs) with cerebral palsy (CP), psychomotor delay, and epilepsy. The Venn diagram displays the number and prevalence rate (\%) of VLBWIs with epilepsy, $\mathrm{CP}$, and psychomotor delay among eligible subjects $(n=$ 8431). In total, 1524 (18.1\%) showed either of these neurological sequelae, while $41(0.5 \%)$ developed all three sequelae by age 3 years

least one of these neurological sequelae by age 3 years. Moreover, 1114 (13.2\%) showed psychomotor delay, 683 (8.1\%) had CP, and 143 (1.7\%) developed epilepsy. Three 
Table 2 Clinical factors associated with epilepsy $(n=143)$

\begin{tabular}{|c|c|c|c|c|c|c|c|c|}
\hline \multirow[b]{2}{*}{ Variables } & \multirow[b]{2}{*}{ Median $/ n$} & \multirow[b]{2}{*}{ Range/\% } & \multicolumn{3}{|c|}{ Crude } & \multicolumn{3}{|c|}{ Adjusted } \\
\hline & & & OR & $95 \% \mathrm{CI}$ & $P$ & OR & $95 \% \mathrm{CI}$ & $P$ \\
\hline${ }^{\mathrm{a}}$ Gestational age [weeks, days] median, range & $29 w 3 d$ & $22 w 0 d-37 w 1 d$ & 0.83 & $0.78-0.88$ & $<0.01$ & 0.89 & $0.60-1.32$ & 0.55 \\
\hline${ }^{a}$ Birth weight $[$ grams] median, range & 870 & $321-1480$ & 0.86 & $0.81-0.91$ & $<0.01$ & 0.98 & $0.95-1.02$ & 0.30 \\
\hline Maternal age of 35 or more years old & 27 & 21.3 & 0.65 & $0.42-1.00$ & 0.05 & 0.48 & $0.20-1.16$ & 0.10 \\
\hline Multiple birth & 41 & 28.7 & 1.19 & $0.83-1.72$ & 0.34 & 1.12 & $0.48-2.59$ & 0.79 \\
\hline Chorioamnionitis & 44 & 30.8 & 1.21 & $0.84-1.73$ & 0.30 & 1.96 & $0.90-4.29$ & 0.09 \\
\hline Antenatal steroid & 60 & 42.0 & 0.85 & $0.61-1.19$ & 0.34 & 0.67 & $0.33-1.35$ & 0.26 \\
\hline Cesarean section & 105 & 73.4 & 0.69 & $0.47-1.00$ & 0.05 & 1.60 & $0.46-1.11$ & 0.46 \\
\hline Male & 89 & 62.2 & 1.56 & $1.11-2.20$ & 0.01 & 1.91 & $0.90-4.02$ & 0.09 \\
\hline Apgar score of less than 7-point at 5-minutes after birth & 40 & 31.8 & 2.09 & $1.43-3.05$ & $<0.01$ & 0.83 & $0.59-1.18$ & 0.92 \\
\hline Small-for-gestational age & 46 & 32.2 & 0.71 & $0.50-1.01$ & 0.05 & 1.45 & $0.40-5.23$ & 0.57 \\
\hline Respiratory distress syndrome & 99 & 69.2 & 1.76 & $1.23-2.52$ & $<0.01$ & 1.50 & $0.53-4.24$ & 0.45 \\
\hline Moderate to severe bronchopulmonary dysplasia & 49 & 66.2 & 1.94 & $1.19-3.15$ & 0.01 & 1.85 & $0.84-4.05$ & 0.12 \\
\hline Sepsis & 26 & 18.2 & 3.04 & $1.97-4.68$ & $<0.01$ & 3.23 & $1.52-6.85$ & $<0.01$ \\
\hline Symptomatic patent ductus arteriosus & 75 & 57.7 & 1.67 & $1.17-2.37$ & $<0.01$ & 0.65 & $0.29-1.42$ & 0.28 \\
\hline Severe intraventricular hemorrhage & 41 & 28.7 & 17.1 & $11.6-25.3$ & $<0.01$ & 5.13 & $2.10-12.5$ & $<0.01$ \\
\hline Cystic periventricular leukomalacia & 46 & 32.2 & 18.3 & $12.6-26.7$ & $<0.01$ & 12.7 & $5.34-30.3$ & $<0.01$ \\
\hline Necrotizing enterocolitis & 6 & 4.2 & 6.79 & $2.87-16.1$ & $<0.01$ & 3.02 & $0.60-15.1$ & 0.18 \\
\hline Treating retinopathy of prematurity & 48 & 33.8 & 2.96 & $2.08-4.21$ & $<0.01$ & 1.74 & $0.85-3.55$ & 0.13 \\
\hline
\end{tabular}

${ }^{\mathrm{a}}$ Continuous variables are expressed as the median and range

Statistically significant $P$-values are in bold italics

hundred and seventy-five (4.4\%) VLBWIs developed two or more of these sequelae in combination (Fig. 2).

\section{Perinatal risk factors associated with epilepsy and other neurological sequelae}

To examine whether VLBWIs with epilepsy had a higher rate of perinatal complications than the whole population of VLBWIs, we performed univariate crude and multivariate adjusted analyses on the same subsets of variables as listed in Table 1. VLBWIs with epilepsy had perinatal history of sepsis (18.2\%), severe IVH (28.7\%), or cystic PVL (32.2\%) at higher frequencies compared with the whole population of VLBWIs $(P<0.01$, Table 2). Cystic PVL showed the highest adjusted OR (AOR) of 12.7 with $95 \%$ CI of 5.34-30.3. Sepsis (AOR 3.23; 95\% CI 1.52-6.85) and severe IVH (AOR 5.13; 95\% CI 2.10-12.5) were also ranked among the leading complications associated with onset of epilepsy in VLBWIs.

\section{Common and unique risk factors for development of epilepsy in VLBWIs}

We next investigated whether these three neonatal complications (cystic PVL, severe IVH, and sepsis) were uniquely identified risk factors for the development of epilepsy. We conducted identical tests for 1524 VLBWIs with psychomotor delay, CP, or epilepsy. Eight of the 18 perinatal variables were associated with the development of one of these three complications (Table S1). As expected from the results of Table 2, 192 (12.6\%) of these VLBWIs showed cystic PVL, 158 (10.4\%) had severe IVH, and 200 (13.2\%) experienced sepsis during the neonatal period (Table S1). Statistical analysis revealed that these three complications were significantly associated with the development of any of the three neurological sequelae by age 3 years $(P<0.01)$.

Demographic analysis further identified $41(0.48 \%)$ VLBWIs who developed all three sequelae in combination by age 3 years (Fig. 2). To gain further insight into their risk factors, we repeated the same analysis for those presenting with all three sequelae in combination, as formerly described (Table S2). A higher proportion of these VLBWIs experienced cystic PVL (39.0\%) and severe IVH (26.8\%) during the neonatal period than those with any of the neurological sequelae $(12.6 \%$ and $10.4 \%$, respectively; Table S1). These data indicated that cystic PVL and severe IVH were the most common and strongest associations for development of epilepsy, psychomotor delay, and CP in VLBWIs.

Lastly, we aimed to establish the unique risk factors for epilepsy. We analyzed further the demographic features of 1524 VLBWIs who showed either CP, psychomotor delay, 
or epilepsy. We subdivided the subjects into those with epilepsy $(n=143)$ and those without epilepsy $(n=1381)$. These two groups showed different comorbidity rates with $\mathrm{CP}$ and psychomotor delay. Compared with the NonEpilepsy Group, the Epilepsy Group had higher comorbidity rates with $\mathrm{CP}(74.1 \%$ vs. $41.8 \%)$ but lower rates of psychomotor delay ( $35.0 \%$ vs. $77.0 \%)$. Both groups showed the highest AORs for development of neurological sequelae when they presented with cystic PVL (12.7 and 4.76) or severe IVH (5.13 and 2.95) (Fig. S1). None of these variables significantly differed in AORs between the two groups.

\section{Discussion}

We clarified development of epilepsy by age 3 years among VLBWIs in Japan and investigated its risk factors. We identified, using the nationwide NRNJ database for VLBWIs, 143 (1.7\%) infants who developed epilepsy. This percentage was higher than that among children in the general population $(0.3-0.6 \%)$ [25, 26], and significantly higher than that in children with epilepsy onset age $<3$ years in Japan $(0.6 \%)$ [27]. This indicates that various causes of brain injury contribute to the onset of epilepsy in preterm-delivered infants [28]. We also found that the prevalence rate was higher than that reported $(1.0 \%)$ in an earlier nationwide cohort from Finland but lower than that reported in Turkey $(5.6 \%)$ [7, 29]. In terms of birth weight, $43.7 \%(n=3685)$ of infants had an extremely low birth weight $<1 \mathrm{~kg}$, and among them, 91 (2.5\%) had epilepsy. This finding suggests that lower birth weight is associated with epilepsy onset by age 3 years in Japan. The results might have varied with differences in subpopulations of severely affected VLBWIs who survived the neonatal period, or with different ethical standards for the management of high-risk infants with multiple complications [26, 30].

Our study identified sepsis, severe IVH, and cystic PVL as the associations of epilepsy in VLBWIs. History of sepsis is also a known risk factor for the development of neonatal seizures and other comorbidities [31]. Among the clinical variables, the association between neonatal seizures and development of epilepsy has been shown in previous studies $[29,32]$. However, we did not include neonatal seizures as one of the clinical variables because of ambiguous diagnoses with or without electroencephalography. Our data confirmed key findings in previous studies and that preterm infants with severe complications are at high risk for persistent neurological sequelae, including epilepsy. In our subjects, the comorbidity rate with psychomotor delay was lower in the Epilepsy Group than that in the Non-Epilepsy Group. Our extensive multivariate analyses further demonstrated that severe IVH and cystic PVL were recurrent risk factors for development of the other two sequelae, $\mathrm{CP}$ and psychomotor delay. This confirms that we were unable to detect unique predisposing factors that were solely associated with onset of epilepsy.

Among 1524 VLBWIs with at least one sequela, 375 (24.6\%) showed more than two. We identified multiple perinatal variables that were overly represented among VLBWIs with psychomotor delay, CP, or epilepsy. Although we applied multivariate analysis to identify explanatory variables, it merely confirmed that severely affected infants were more likely to show unfavorable neurological outcomes than were those with milder distress at birth [33]. It remains to be clarified whether these risk factors place differential weight on neurological outcomes of VLBWIs. Therefore, a scoring system will provide clues for characterizing more accurately those with a risk of developing epilepsy, in addition to other neurodevelopmental sequelae [34].

The strength of our study was that we used a nationwide cohort delineating the neurodevelopmental profiles for a whole population of VLBWIs in Japan over a 10-year period. To provide comprehensive data from eligible subjects, we aimed to determine the optimal age to investigate their neurodevelopmental profiles. By setting it to 3 years, we clarified that $8.2 \%$ and $14.9 \%$ of VLBWIs $(n=8431)$ developed CP and psychomotor delay, respectively, and we regard these results as reasonable. We considered that 3 years was an appropriate age at which to evaluate neurological comorbidities because there were too-high drop-off rates at older ages. In contrast, we understand that neurodevelopmental deficits might further evolve in preschool and school-age children [35]. More accurate profiles in neurocognitive functions of VLBWIs will therefore be unveiled through pursuing their educational performance and achievements.

We recognize several limitations to the present study. First, the follow-up rate was only 54.8\% among 15,471 potentially eligible subjects. In Japan, there is a traditional perinatal support system for a pregnant woman. She returns to her parents' home before labor, delivers at a hospital near her hometown, and the baby grows up with parental support. Meanwhile, a follow-up study is conducted by a doctor at the NICU where each infant was under medical care. In cases where the woman has moved from her hometown after hospital discharge, researchers could be at a risk of missing the subject. Many pregnant women choose this practice of delivery in their hometown, and their infants are raised in the NICU away from the place where they will grow up afterward. Thus, the NRNJ studies have had a high percentage (43-63\%) of drop-outs [22-24]. We did not choose earlier ages for evaluation because too short a duration of follow-up may have compromised the accuracy 
of estimating the prevalence of children with epilepsy. We therefore considered that our data fully disclosed the neurological features of VLBWIs with the currently available dataset from the NRNJ database. In addition, there was a lack of data for CP and developmental delay in the excluded population. In those without epilepsy data $(n=6912)$, only 2090 had data for CP, and only 1897 had data for psychomotor delay. Consequently, the study population may not be representative of the whole population. The percentages of sepsis and IVH were significantly different between the patients with and without epilepsy data. Those two variables implied a possibility to deviate the analysis in our study; however, we were not able to evaluate whether the study population had more severe disability than the excluded group. The other limitation was the lack of detailed information about epilepsy: age of onset, diagnostic categories, medication, and clinical course of epileptic seizures. Their correlations with findings in electroencephalography or neuroimaging were not documented either. These data will further characterize the nature of pathogenic insults to the brain of VLBWIs.

In conclusion, the NRNJ database revealed that severe IVH, cystic PVL, and sepsis were critical associations for the onset of epilepsy in VLBWIs by age 3 years. Severe IVH and cystic PVL were also associated with the development of other sequelae, CP and psychomotor delay. These data will serve as a reference for neonatologists to identify therapeutic targets and improve outcomes of neurological morbidity in high-risk VLBWIs [21]. With regard to epilepsy specific for VLBWIs, details of its characterization, onset age, classification, and severity should be described in a future study.

Acknowledgements We thank Cathel Kerr, BSc, PhD, from Edanz Group (www.edanzediting.com/ac) for editing a draft of this papert. The Neonatal Research Network of Japan consisted of the following institutions; Sapporo City General Hospital, Asahikawa Kosei General Hospital, Engaru-Kosei General Hospital, Kushiro Red Cross Hospital, Obihiro-Kosei General Hospital, Tenshi Hospital, NTT Higashinihon Sapporo Hospital, Nikko Memorial Hospital, Nayoro City General Hospital, Sapporo Medical University, Asahikawa Medical University, Aomori Prefectural Central Hospital, Iwate Medical University, Iwate Prefectural Ofunato Hospital, Iwate Prefectural Kuji Hospital, Iwate Prefectural Ninohe Hospital, Sendai Red Cross Hospital, Akita Red Cross Hospital, Tsuruoka Municipal Shonai Hospital, Yamagata University, Yamagata Prefectural Central Hospital, Fukushima Medical University, Takeda General Hospital, Fukushima National Hospital, Tsukuba University, Tsuchiura Kyodo Hospital, Ibaraki Children's Hospital, Dokkyo Medical University, Jichi Medical University, Ashikaga Red Cross Hospital, Gunma Children's Medical Center, Kiryu Kosei General Hospital, Fuji Heavy Industries Health Insurance Society Ota Memorial Hospital, Gunma University, Saitama Children's Medical Center, Nishisaitama-chuo National Hospital, Saitama Medical University Saitama Medical Center, Kawaguchi Municipal Medical Center, Jichi Medical University Saitama Medical Center, Asahi General Hospital, Chiba Kaihin Municipal Hospital, Kameda Medical Center, Tokyo Women's Medical University Yachiyo Medical Center, Juntendo University Urayasu
Hospital, Tokyo Metropolitan Children's Medical Center, Tokyo Women's Medical University, Aiiku Hospital, Nihon University Itabashi Hospital, National Center for Global Health and Medicine, Tokyo Medical University, Teikyo University, Showa University, Japan Red Cross Medical Center, National Center for Child Health and Development, Tokyo Metropolitan Otsuka Hospital, Toho University, Tokyo Metropolitan Bokuto Hospital, Tokyo Jikei Medical University, Tokyo Medical and Dental University, Saint Luku's International Hospital, Juntendo University, Sanikukai Hospital, Katsushika Red Cross Hospital, Yokohama Rosai Hospital, Yokohama City University Medical Center, St. Marianna University School of Medicine Hospital, Kanagawa Children's Medical Center, Tokai University, Kitazato University, Odawara Municipal Hospital, Nippon Medical School Musashi Kosugi Hospital, Saiseikai Yokohamashi Tobu Hospital, National Hospital Organization Yokohama Medical Center, Yamanashi Prefectural Central Hospital, Nagano Children's Hospital, Shinshu University, Iida Municipal Hospital, National Hospital Organization Shinshu Ueda Medical Center, Saku General Hospital, Niigata University, Niigata Prefectural Central Hospital, Niigata Municipal Hospital, Nagaoka Red Cross Hospital, Koseiren Takaoka Hospital, Toyama Prefectural Central Hospital, Toyama University, Ishikawa Medical Center for Maternal and Child Health, Kanazawa Medical University, Kanazawa Medical Center, Fukui Prefectural Hospital, Fukui University, Gifu Prefectural General Medical Center, National Hospital Organization Nagara Medical Center, Takayama Red Cross Hospital, Seirei Hamamatsu Hospital, Shizuoka Saiseikai Hospital, Shizuoka Children's Hospital, Hamamatsu Medical University, Numazu Municipal Hospital, Yaizu City Hospital, Fujieda Municipal General Hospital, Nagoya Red Cross Daini Hospital, Nagoya University, Nagoya Red Cross Daiichi Hospital, Toyohashi Municipal Hospital, Nagoya City West Medical Center, Anjo kosei Hospital, Tosei General Hospital, Komaki Municipal Hospital, Toyota Memorial Hospital, Okazaki Municipal Hospital, Konan Kosei Hospital, National Mie Central Medical Center, Ise Red Cross Hospital, Yokkaichi Municipal Hospital, Otsu Red Cross Hospital, Shiga University of Medical Science Hospital, Nagahama Red Cross Hospital, Uji Tokushukai Hospital, The Japan Baptist Hospital, Kyoto University, Kyoto Red Cross Daiichi Hospital, National Maizuru Medical Center, Fukuchiyama City Hospital, Kyoto Prefectural University of Medicine Hospital, Kyoto City Hospital, Mitsubishi Kyoto Hospital, Yodogawa Christian Hospital, Osaka Medical Center and Research Institute for Maternal and Child Health, Osaka University, Takatsuki General Hospital, Kansai Medical University, Osaka City General Hospital, Osaka City Sumiyoshi Hospital, Aizenbashi Hospital, Toyonaka Municipal Hospital, National Cerebral and Cardiovascular Center, Kitano Hospital, Saiseikai Suita Hospital, Chifune Hospital, Bell-land General Hospital, Rinku General Medical Center, Osaka Red Cross Hospital, Yao Municipal Hospital, Osaka General Medical Center, Osaka City University, Hyogo Prefectural Kobe Children's Hospital, Kobe University, Kakogawa West City Hospital, Saiseikai Hyogoken Hospital, Kobe City Medical Center General Hospital, Hyogo College of Medicine Hospital, Himeji Red Cross Hospital, Toyooka Public Hospital, Hyogo Prefectural Awaji Medical Center, Nara Medical University, Wakayama Medical University, Tottori Prefectural Central Hospital, Tottori University, Shimane Prefectural Central Hospital, Matsue Red Cross Hospital, Kurashiki Central Hospital, Tsuyama Central Hospital, Kawasaki Medical School Hospital, National Hospital Organization Okayama Medical Center, Okayama Red Cross Hospital, Hiroshima City Hiroshima Citizens Hospital, Hiroshima Prefectural Hospital, Hiroshima University, Tsuchiya General Hospital, National Hospital Organization Kure Medical Center, Yamaguchi University, Yamaguchi Grand Medical Center, Tokushima University, Tokushima Municipal Hospital, Kagawa University, National Hospital Organization Kagawa Children's Hospital, Matsuyama Red Cross Hospital, Ehime Prefectural Central Hospital, Kochi Health Science Center, St. Mary's Hospital, National Kyushu Medical Center, 
Kurume University, Kitakyushu Municipal Medical Center, University of Occupational and Environmental Health, Fukuoka University, Kyushu University, Iizuka Hospital, National Hospital Organization Kokura Medical Center, National Hospital Organization Saga Hospital, National Hospital Organization Nagasaki Medical Center, Kumamoto City Hospital, Kumamoto University, Oita Prefectural Hospital, Almeida Memorial Hospital, Nakatsu Municipal Hospital, Miyazaki University, National Hospital Organization Miyakonojo Medical Center, Kagoshima City Hospital, Imakiire General Hospital, Okinawa Prefectural Nanbu Medical Center \& Children's Medical Center, Okinawa Prefectural Chubu Hospital, Naha City Hospital, Okinawa Red Cross Hospital.

Funding This study was supported in part by JSPS KAKEN Grant Number JP17K16268 (YM), JP17K16301 (MT), JP17K16300 (HI) and JP16K19688 (JF).

\section{Compliance with ethical standards}

Conflict of interest The authors declare that they have no conflict of interest.

Ethics This study was approved by the Institutional Review Board of Tokyo Women's Medical University and registered as a prospective observational study with the University Hospital Medical Information Network (UMIN000006961). Written informed consent to the research and publication of the results was obtained from the parents or guardians of all infants.

Publisher's note Springer Nature remains neutral with regard to jurisdictional claims in published maps and institutional affiliations.

Open Access This article is licensed under a Creative Commons Attribution 4.0 International License, which permits use, sharing, adaptation, distribution and reproduction in any medium or format, as long as you give appropriate credit to the original author(s) and the source, provide a link to the Creative Commons license, and indicate if changes were made. The images or other third party material in this article are included in the article's Creative Commons license, unless indicated otherwise in a credit line to the material. If material is not included in the article's Creative Commons license and your intended use is not permitted by statutory regulation or exceeds the permitted use, you will need to obtain permission directly from the copyright holder. To view a copy of this license, visit http://creativecommons. org/licenses/by/4.0/.

\section{References}

1. Younge N, Goldstein RF, Bann CM, Hintz SR, Patel RM, Smith $\mathrm{PB}$, et al. Survival and neurodevelopmental outcomes among periviable infants. N Engl J Med. 2017;376:617-28.

2. Kusuda S, Fujimura M, Uchiyama A, Totsu S, Matsunami K, Neonatal Research Network. Trends in morbidity and mortality among very-low-birth-weight infants from 2003 to 2008 in Japan. Pediatr Res. 2012;72:531-8.

3. Inoue H, Ochiai M, Yasuoka K, Tanaka K, Kurata H, Fujiyoshi J, et al. Early mortality and morbidity in infants with birth weight of 500 grams or less in Japan. J Pediatr. 2017;190:112. e113

4. Patel RM. Short- and long-term outcomes for extremely preterm infants. Am J Perinatol. 2016;33:318-27.

5. Kono Y, Mishina J, Yonemoto N, Kusuda S, Fujimura M. Outcomes of very-low-birthweight infants at 3 years of age born in 2003-2004 in Japan. Pediatr Int. 2011;53:1051-8.
6. Sun Y, Vestergaard M, Pedersen CB, Christensen J, Basso O, Olsen J. Gestational age, birth weight, intrauterine growth, and the risk of epilepsy. Am J Epidemiol. 2008;167:262-70.

7. Koc O, Kavuncuoglu S, Ramoglu MG, Aldemir E, Aktalay A, Eras Z. School performance and neurodevelopment of very low birth weight preterm infants: first report from Turkey. J Child Neurol. 2016;31:170-6.

8. Crump C, Sundquist K, Winkleby MA, Sundquist J. Preterm birth and risk of epilepsy in Swedish adults. Neurology. 2011;77:1376-82.

9. Kusuda S, Fujimura M, Uchiyama A, Nakanishi H, Totsu S, Neonatal Research Network. Identification of practices and morbidities affecting the mortality of very low birth weight infants using a multilevel logistic analysis: clinical trial or standardisation?. BMJ Open. 2013;3:5

10. Maruyama H, Yonemoto N, Kono Y, Kusuda S, Fujimura M, Neonatal Research Network of Japan. Weight growth velocity and neurodevelopmental outcomes in extremely low birth weight infants. PloS ONE. 2015;10:12

11. Linsell L, Malouf R, Morris J, Kurinczuk JJ, Marlow N. Prognostic factors for cerebral palsy and motor impairment in children born very preterm or very low birthweight: a systematic review. Dev Med Child Neurol. 2016;58:554-69.

12. Tita AT, Andrews WW. Diagnosis and management of clinical chorioamnionitis. Clin Perinatol. 2010;37:339-54.

13. Itabashi $K$, Miura F, Uehara R, Nakamura $Y$. New Japanese neonatal anthropometric charts for gestational age at birth. Pediatr Int. 2014;56:702-8.

14. Ehrenkranz RA, Walsh MC, Vohr BR, Jobe AH, Wright LL, Fanaroff AA, et al. Validation of the National Institutes of Health consensus definition of bronchopulmonary dysplasia. Pediatrics. 2005; 116:1353-60.

15. Papile LA, Burstein J, Burstein R, Koffler H. Incidence and evolution of subependymal and intraventricular hemorrhage: a study of infants with birth weights less than 1,500 gm. J Pediatr. 1978;92:529-34.

16. Bell MJ, Ternberg JL, Feigin RD, Keating JP, Marshall R, Barton L, et al. Neonatal necrotizing enterocolitis. Therapeutic decisions based upon clinical staging. Ann Surg. 1978;187:1-7.

17. Members of an International Classification of Retinopathy of Prematurity Australia. The international classification of retinopathy of prematurity revisited. Arch Ophthalmol. 123:991-9.

18. Tamaru S, Kikuchi A, Takagi K, Wakamatsu M, Ono K, Horikoshi T, et al. Neurodevelopmental outcomes of very low birth weight and extremely low birth weight infants at 18 months of corrected age associated with prenatal risk factors. Early Hum Dev. 2011;87:55-9.

19. BaxMC. Terminology and classification of and classification of cerebral palsy. Dev Med Child Neurol. 1964;6:295-7.

20. Chang BS, Lowenstein DH. Epilepsy. N Engl J Med. 2003;349: 1257-66.

21. Isayama $T, Y e X Y$, Tokumasu $H$, Chiba $H$, Mitsuhashi $H$, Shahrook $\mathrm{S}$, et al. The effect of professional-led guideline workshops on clinical practice for the management of patent ductus arteriosus in preterm neonates in Japan: a controlled before-andafter study. Implement Sci. 2015;10:67.

22. Kimura T, Takeuchi M, Imai T, Tanaka S, Kawakami K, Neonatal Research Network of Japan. Neurodevelopment at 3 years in neonates born by vaginal delivery versus cesarean section at $<26$ weeks of gestation: retrospective analysis of a nationwide registry in Japan. Neonatology. 2017;112:258-66.

23. Inoue H, Ochiai M, Sakai Y, Yasuoka K, Tanaka K, Ichiyama M, et al. Neurodevelopmental outcomes in infants with birth weight $\leq 500 \mathrm{~g}$ at 3 years of age. Pediatrics. 2018;142:e20174286. https:// doi.org/10.1542/peds.2017-4286. Epub 16 Nov 2018.

24. Nakanishi H, Suenaga H, Uchiyama A, Kusuda S, Neonatal Research Network of Japan. Persistent pulmonary hypertension of 
the newborn in extremely preterm infants: a Japanese cohort study. Arch Dis Child Fetal Neonatal Ed. 2018;103: F554-61.

25. Russ SA, Larson K, Halfon N. A national profile of childhood epilepsy and seizure disorder. Pediatrics. 2012;129:256-64.

26. Chiang KL, Cheng CY. Prevalence and neuro-psychiatric comorbidities of pediatric epilepsy in Taiwan: a national population-based study. Epilepsy Res. 2014;108:1451-60.

27. Oka E. Childhood epilepsy in Okayama Prefecture, Japan-a neuroepidemiological study. Brain Dev. 2002;34:95-102.

28. Douglass LM, Heeren TC, Stafstrom CE, DeBassio W, Allred EN, Leviton A, et al. Cumulative incidence of seizures and epilepsy in ten-year-old children born before 28 weeks' gestation. Pediatr Neurol. 2017;73:13-9.

29. Hirvonen M, Ojala R, Korhonen P, Haataja P, Eriksson K, Gissler M, et al. The incidence and risk factors of epilepsy in children born preterm: a nationwide register study. Epilepsy Res. 2017;138:32-8.
30. Kohelet D, Shochat R, Lusky A, Reichman B. Risk factors for seizures in very low birthweight infants with periventricular leukomalacia. J Child Neurol. 2006;21:965-70.

31. Vesoulis ZA, Inder TE, Woodward LJ, Buse B, Vavasseur C, Mathur AM. Early electrographic seizures, brain injury, and neurodevelopmental risk in the very preterm infant. Pediatr Res. 2014;75:564-9.

32. Soltirovska-Salamon A, Neubauer D, Petrovcic A, Paro-Panjan D. Risk factors and scoring system as a prognostic tool for epilepsy after neonatal seizures. Pediatr Neurol. 2014;50:77-84.

33. Kidokoro H, Anderson PJ, Doyle LW, Woodward LJ, Neil JJ, Inder TE. Brain Injury and altered brain growth in preterm infants: predictors and prognosis. Pediatrics. 2014;134:E444-53.

34. Pisani F, Sisti L, Seri S. A scoring system for early prognostic assessment after neonatal seizures. Pediatrics. 2009;124: e580-7.

35. Moster D, Lie RT, Markestad T. Long-term medical and social consequences of preterm birth. N Engl J Med. 2008;359:262-73. 